\title{
Nueva opción terapéutica para el tratamiento de la insuficiencia cardiaca
}

\author{
A new therapeutic option for heart failure patients
}

\section{Objetivos}

Comparar la eficacia de la asociación de un bloqueante de los receptores de angiotensina más un inhibidor de la neprilisina (LCZ696) versus enalapril en pacientes con insuficiencia cardíaca (IC) y fracción de eyección deprimida.

\section{Diseño}

Ensayo clínico controlado aleatorizado doble ciego. El estudio comprendió tres fases sucesivas: 1) de rastreo, 2) de prueba o run-in (en la que los pacientes recibieron de manera simple ciego enalapril seguido por LCZ696) para asegurar una aceptable tolerancia a las drogas, 3 ) de asignación aleatoria de las intervenciones (doble ciego).

\section{Lugar}

Estudio multicéntrico multinacional en el que participaron centros de Norte América, Latinoamérica, Europa y Asia.

\section{Pacientes}

Fueron incluidos 8442 pacientes con IC (CF II-III o IV) con disminución de la fracción de eyección del ventrículo izquierdo (inicialmente $<40 \%$ y luego de una enmienda del protocolo $<35 \%)$. Para el diagnóstico de IC, además de los datos clínicos
McMurray J y col. N Engl J Med 2014;371:993-1004 se exigió un valor elevado de BNP o pro-BNP ( $\geq 150$ y $\geq 600$ $\mathrm{pg} / \mathrm{mL}$ respectivamente). Fueron excluidos los pacientes con presión arterial sistólica $<100 \mathrm{~mm} \mathrm{Hg}$ (Kalemia $>5,2 \mathrm{Meq} / \mathrm{L} \mathrm{o}$ clearence de Creatinina $<30 \mathrm{~mL} / \mathrm{min} / 1,73 \mathrm{~m}^{2}$ ).

\section{Intervención}

Los pacientes recibieron dos veces por día LCZ696 200 mg $(n=4187)$ o enalapril $10 \mathrm{mg}(n=4212)$. LCZ96 incluía un bloqueante de los receptores de angiotensina, equivalente a 160 mg de valsartan y sacubitril, un inhibidor de la neprilisina.

\section{Medición de resultados principales}

El punto final primario combinado incluyó mortalidad cardiovascular y hospitalizaciones por IC. Otros puntos finales fueron las hospitalizaciones por IC (HIC) analizadas en forma aislada y la mortalidad por cualquier causa.

\section{Resultados principales}

LCZ696 fue superior al enalapril para reducir el riesgo de muerte y de HIC. Ver tabla 1. El análisis de subgrupos preespecificado documentó que en todos los casos existía una tendencia o un beneficio claro a favor del LCZ696.

Tabla 1: comparación de la eficacia de LCZ696 y enalapril en pacientes con insuficiencia cardíaca a 27 meses de seguimiento medio.

\begin{tabular}{l|c|c|c|c} 
& LCZ696 (\%) & Enalapril (\%) & HR (IC95\%) & NNT (IC95\%) \\
\hline Punto final primario & $914(21,8 \%)$ & $914(21,8 \%)$ & $0,80(0,73 \mathrm{a} 0,87)$ & $21(15 \mathrm{a} 34)$ \\
\hline Muerte cardiovascular & $558(13,3 \%)$ & $558(13,3 \%)$ & $0,80(0,71 \mathrm{a} 0,89)$ & $32(22 \mathrm{a} 63)$ \\
\hline Hospitalización por IC & $537(12,8 \%)$ & $537(12,8 \%)$ & $0,79(0,71 \mathrm{a} 0,89)$ & $36(23 \mathrm{a} 77)$ \\
\hline Muerte & $711(17,0 \%)$ & $711(17,0 \%)$ & $0,84(0,76 \mathrm{a} 0,93)$ & $35(22 \mathrm{a} 83)$ \\
\hline
\end{tabular}

IC: insuficiencia cardíaca

\section{Conclusiones}

LCZ696 fue superior al enalapril para reducir el riesgo de muerte y hospitalización por IC.
Fuente de financiamiento: Laboratorio Novartis.

\section{Comentario}

Los betabloqueantes, los inhibidores de la enzima convertidora de la angiotensina (IECA) y los antialdosterónicos se aceptan como pilares para disminuir la mortalidad de los pacientes con IC crónica con función ventricular disminuida $a^{1,2,3}$. Los bloqueantes de los receptores de la angiotensina sólo se usan cuando no se toleran Ios IECA y su efecto se manifiesta fundamentalmente en la reducción de las hospitalizaciones ${ }^{4}$. El beneficio de la ivabradina aprobada por el ente europeo y recientemente por la Administración de Drogas y Alimentos de EE.UU. (FDA) se limita a los pacientes con frecuencia cardíaca superior a 70 latidos/minutos y ha mostrado reducir el número de las hospitalizaciones ${ }^{5}$. Por lo tanto, la combinación terapéutica planteada en este estudio representa un aporte luego de varios años de estancamiento en el tema, ya que redujo significativamente la mortalidad cardiovascular y las reinternaciones por IC con respecto al enalapril ${ }^{6}$. Los inhibidores de la neprilisina fueron probados en un estudio que debió suspenderse por la alta frecuencia de angiodema en el grupo omapatrilat ${ }^{7}$. El sacubitril con respecto al omapatrilat tiene propiedades farmacológicas distintas sobre otros grupos enzimáticos que degradan bradiquininas y en el estudio actual, la frecuencia de angiodema fue mínima (1/1000) y similar en ambos grupos ${ }^{8}$. La población que integra el estudio representa una población promedio de los estudios de IC, aunque la edad promedio de 64 años está por debajo de la de los registros de esta patología. Por otro lado, más del $90 \%$ de los pacientes recibían betabloqueantes y más del $50 \%$, antialdosterónicos, o sea que estaban aceptablemente medicados. Respecto del punto final primario, fue el utilizado por la mayoría de los estudios de IC y el tamaño muestral fue estimado anticipando una mortalidad cardiovascular de $7 \%$ por año para el grupo control. El promedio de seguimiento fue de 27 meses y la pérdida de seguimiento fue despreciable. La detención prematura del estudio respondió a causas pre determinadas por el protocolo. En síntesis se respetaron muchas de las condiciones esperadas para un estudio clínico controlado aleatorizado doble ciego. La única duda que plantea el estudio es la de haber empleado un doble run-in. Los pacientes debían tolerar 20 $\mathrm{mg} / \mathrm{día}$ de enalapril (durante un promedio de semanas promedio) y luego $400 \mathrm{mg} / \mathrm{día}$ de LCZ696 (por 4 semanas) y los que no presentaban efectos adversos inaceptables, eran aleatorizados. El $12 \%$ de los pacientes no toleraron la etapa de runin y como la etapa bajo LCZ696 fue más prolongada, podría interpretarse como una menor frecuencia de intolerancia a la droga en estudio que al enalapril. Sin embargo, no se puede saber cuál hubiera sido la frecuencia de intolerancia si el run-in 
hubiera comenzado con LCZ696. Además, la frecuencia de hipotensión arterial fue significativamente mayor en el grupo LCZ696 que en el grupo enalapril. Este doble filtro pre-aleatorización, inexorablemente seleccionó a la población más tolerante a las drogas y podría limitar la validez externa del estudio.

\section{Conclusiones del comentador}

Luego de muchos años se publica una opción terapéutica que reduce la mortalidad en los pacientes con IC de manera consistentes en todos los subgrupos analizados. El diseño seleccionó a los pacientes que toleran tanto el enalapril como el LCZ696, por lo que persiste la duda de su generalización a otros pacientes con IC.

César Belziti [ Servicio de Cardiología, Unidad de Insuficiencia Cardíaca del Instituto de Medicina Cardiovascular del Hospital Italiano de Buenos Aires. cesar.belziti@ hospitalitaliano.org.ar]

Belzitti C. Nueva opción terapéutica para el tratamiento de la insuficiencia cardíaca. Evid Act Pract Ambul. 2016:19(1):13-14. Ene-Mar. Comentado de: McMurray JJ, y col. Angiotensin-neprilysin inhibition versus enalapril in heart failure. NEJM. 2014;371(11):993-1004. PMID: 25176015.

\section{Referencias:}

1. Packer M y col. The effect of carvedilol on morbidity and mortality in patients with chronic heart failure. N Engl J Med 1996;334:1349-55.

2. The CONSENSUS Trial Study Group. Effects of enalapril on mortality in severe

congestive heart failure: results of the Cooperative North Scandinavian Enalapril Survival Study (CONSENSUS). N Engl J Med 1987:316:1429-35

3. Pitt B y col. The effect of spironolactone on morbidity and mortality in patients with severe heart failure. N Engl J Med 1999;341:709-17.

4. Zannad Fy col. Eplerenone in patients with systolic heart

failure and mild symptoms. N Engl J Med2011:364:11-21.

5. Young $\mathrm{J}$ y col. Mortality and morbidity reduction with candesartan in patients with chronic heart failure and left ventricular systolic dysfunction: results of the CHARM low left ventricular ejection fraction trials. Circulation 2004;110:2618-26.

5. Swedberg K y col. Ivabradine and outcomes in chronic heart failure (SHIFT): a randomised placebocontrolled study. Lancet 2010;376:875-85. [Erratum, Lancet 2010;376:1988.] 6. John J y col. Desai et al. for the PARADYGM-HF Investigators and Committees. Angiotensin-Neprilysin Inhibition versus Enalapril in Heart Failure. N Engl J Med 2014; 371:993-1004

7. Packer M y col. Comparison of omapatrilat and enalapril in patients with chronic heart failure: the Omapatrilat Versus Enalapril Randomized Trial of Utility in Reducing Events (OVERTURE). Circulation2002;106:920-6.

8. Hegde L y col. Concomitant angiotensin AT1 receptor antagonism

and neprilysin inhibition produces omapatrilat-like anthypertensive effects without promoting tracheal plasma extravasation in the rat. J Cardiovasc Phamacol 2011:57:495-504.

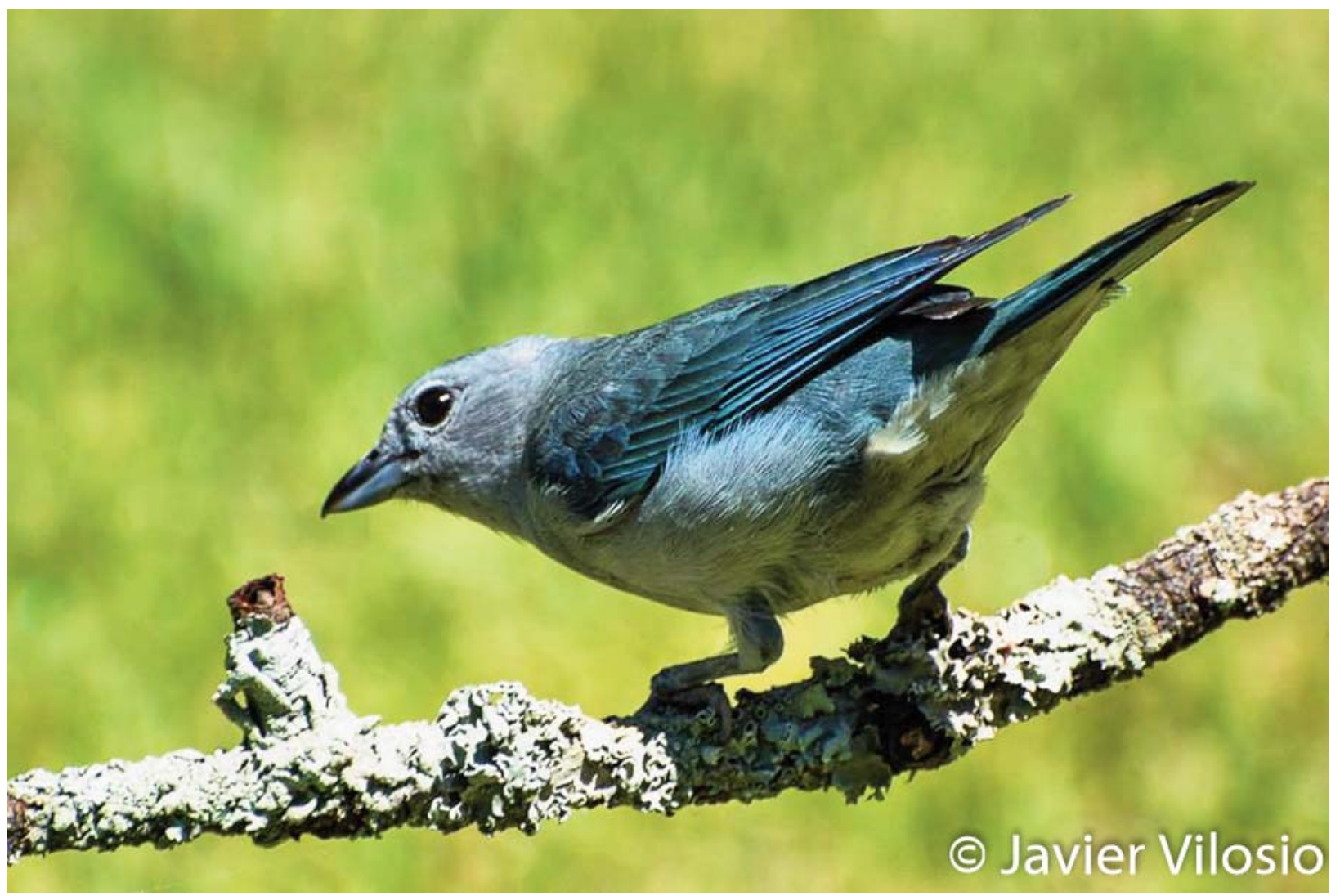

Fotografía: Javier Vilosio 